\title{
The first six years of surveillance in pediatric and neonatal intensive care units in Turkey
}

\author{
Emine Alp', Tülay Orhan², Cemile Atalay Kürkcü², Safiye Ersoy² and Mary-Louise McLaws ${ }^{3 *}$
}

\begin{abstract}
Background: Patients in resourced-limited neonatal and pediatric intensive care units (NICU and PICU) are vulnerable to healthcare associated infections (HAl). We report the incidence of HAl, multidrug resistant microorganisms (MDROs) and the pattern of antibiotic usage in the first six years of a surveillance program in a teaching hospital in Turkey.

Methods: Between 2007 and 2012 surveillance data for HAl, MDROs and antibiotic usage were collected from the infection control department, pathology, hospital admissions and pharmacy. In 2009 hand hygiene auditing was introduced. Hand sanitizer usage was expressed as liters per 1000 patient-days. Antibiotic usage was presented as defined daily doses (DDD). Evidence of change in the incidence of HAl was tested using Poison regression modeling.
\end{abstract}

Results: The rate of gram negative MDRO in PICU increased significant between 2007 and 2012 (IRR 1.5, P=0.033) but remained unchanged in NICU $(P=0.824)$. By 2012 ceftriaxone prescribing in PICU had decreased while carbapenem prescribing increased by $80 \%$. In NICU carbapenem decreased by $42 \%$ and betalactam decreased by $29 \%$. Hand hygiene compliance significantly improved in PICU (IRR 1.9, $p<0.001$ ) and NICU (IRR 2.2, $p<0.001$ ) but compliance remained modest after three years with inconsistent levels across the 5 moments.

Conclusion: The early years of our infection control program highlights the endemicity of HAI and MDROs in our $\mathrm{NICU}$ and PICU. The consistent pattern of antibiotic usage, endemic MROs in PICU and modest hand hygiene clearly provide strategic focuses for intervention.

Keywords: Surveillance, Healthcare, Associated, Infection, Antibiotic, Consumption, Multiple, Resistance

\section{Introduction}

Healthcare associated infections (HAI) remain a major safety problem for vulnerable patients in pediatric and neonatal intensive care units (ICUs) worldwide [1-3]. In low to middle income countries where infection control programs are resource-poor or limited, pediatric ICU (PICU) and neonatal ICU (NICU) patients are especially vulnerable to HAIs [1]. In high-income countries evidence-based infection control programs have successfully reduced HAIs rates [4]. However, the feasibility of successful implementation of programs outside high resourced countries is questionable given the limited data from low and middle income countries [2]. Turkey

\footnotetext{
* Correspondence: m.mclaws@unsw.edu.au

${ }^{3}$ School of Public Health and Community Medicine, UNSW Medicine, The University of New South Wales, Level 3 Samuels Building, Sydney, NSW 2052, Australia

Full list of author information is available at the end of the article
}

is a middle-income country [5] with rising HAI rates and in 2000 the newly formed Turkish Society of Hospital Infection and Control developed guidelines and introduced training for infection control nurses and doctors. The Ministry of Health responded in 2005 by mandating public hospitals provide routine reports on infection control activities and in 2006 a new national surveillance system was rolled out [6]. We report the incidence of HAI over the first 6-years of a surveillance program in a typical tertiary PICU and NICU in Turkey.

\section{Methods \\ Description of institution and infection control program \\ Erciyes University Hospital is a tertiary referral facility in the Central Anatolian region of Turkey. Institutional ap- proval was given for reporting the data. The pediatric hospital is collocated on the adult hospital campus with}


154,680 pediatric admissions and 1,653 ICU admissions annually. Surgery is rarely performed with approximately 11 pediatric or neonatal patients undergoing surgery per year. There are 2 level-III ICUs; a 10-bed pediatric ICU (PICU) for patients aged 1 month to 18 years and an 18bed neonatal ICU (NICU) with babies as small as $<750 \mathrm{~g}$. Bed spacing is $>1.5 \mathrm{~m}$ and there is 1 sink provided for every 2-beds. PICU has 1 isolation room for every 5-beds and in NICU there is 1 isolation room for every 9-beds. In 2006 an organized infection control program introduced a policy that one trained infection control doctor and one certified infection control nurses (ICN) were to be employed for each unit as a full-time equivalent (FTE) staff. Since 2006, all patients admitted to ICU have been routinely screened for vancomycin resistant enterococci and carbapenem resistant Enterobacteriaceae. Surveillance definitions were applied in accordance with the Centers for Disease and Control (CDC) [4]. From 2009 a multimodal hand hygiene education promotion campaigns was introduced that included alcohol based hand rub (ABHR) at every bedside, chlorhexidine containing soap and hand hygiene compliance audits with feedback. Trained ICNs audit healthcare workers' (HCWs) compliance with My Five Moments using the World Health Organization audit tool and audit in accordance with the guidelines [7]. Monthly and quarterly HAI and hand hygiene results are reported to the infection control committee.

\section{Data collection}

Between 2007-2012 surveillance for HAI included active surveillance by the ICN who also validated all laboratory HAI notifications. Surveillance data are collected routinely as a quality and safety activity of the Hospital. These data were collected from the infection control department as well as device utilization and deviceassociated infection rates for central line associated bacteremia (CLABSI), catheter associated urinary tract infections (CAUTI), incidence of multidrug resistant microorganisms (MDRO) and hand hygiene compliance. Hospital admissions provided the number of patient days, occupancy rate per year, length of stay and the 24-h nurse-to-patient ratio. The nursing workload was measured by the 24-h nurse-to-patient ratio divided by the total number of nurses during the 24-h period using the patients' census for the day. Consumption of alcohol based hand rub (ABHR) was provided by pharmacy and expressed as liters per 1000 patient-days. Pharmacy provided antimicrobial use (reported as defined daily dose [DDD] and is the average maintenance dose per day for each drug) normalized per 1000 patient-days in accordance with World Health Organization (WHO Collaborating Centre for Drug Statistics Methodology, 2012). The most widely used antibiotics examined for usage were: ceftriaxone, beta-lactam/beta-lactamase inhibitors, carbapenem, aminoglycoside, glycopeptide, linezolid, metronidazole, quinolones, colistin, tigecycline, fluconazole. A microorganism was defined as MDRO if it provided resistant to more than three classes of antibiotics; aminoglycosides, antipseudomonal penicillins, carbapenems, cephalosporins, beta-lactam/beta-lactamase inhibitor, quinolones, colistin, tigecycline [7]. The most prevalent MDRO included Acinetobacter baumannii, Pseudomonas aeruginosa, extended spectrum beta-lactamase (ESBL) Klebsiella pneumoniae and ESBL Esherichia coli, methicillin resistant Staphylococcus aureus (MRSA) and ampicillin resistant Enterococcus. Pathogens were identified using Vitek-2 automated method and interpreted according to Clinical Laboratory Standards Institute Guidelines: CLSI. Mean inhibitory concentrations were performed using a standard method for antimicrobial susceptibility testing in accordance with Twenty-fourth informational supplement M100-S18 (Villanova, PA, USA, 2008).

\section{Analysis}

The difference in the mean length of stay, with \pm one standard deviation (SD), was calculated using ANOVA. Frequencies and $95 \%$ CIs were established separately for hand hygiene compliance in PICU and NICU from 2009 to 2012. Total HAI rates expressed per 1000 patientdays and VAP, CLABSI and CAUTI were expressed per 1000 device-days, with 95 \% CIs for 2007 to 2012. Exact binomial method around a proportion was used to test for change in incidence. All three specific HAIs were aggregated to examine whether small number of infections in each of the three types prevented detection in the change in rates. On examination of the precision of the estimates, using $95 \%$ CIs, precision was not warranted to one decimal place and all decimals were rounded up at 0.6 and only rates $<1$ were present to one decimal point. Changes in the percentage point difference in hand hygiene rates from 2009 to 2012 were calculated. The effect of the introduction of the My five moments for hand hygiene campaign on total compliance for each of the five hand hygiene indications and HAI was estimated using incidence rate ratio (IRR) produced from a Poisson generalized linear model (GLM) which was chosen because total HAI had a Poisson distribution. IRR could not be established where rates were zero. The first year of observation was used as the baseline for measuring change in IRRs over the following years. HAI were statistically rare events resulting in the inability for the effect of hand hygiene on specific HAIs to be modeled using either GLM or negative binomial regression. Hand hygiene compliance rates were also reported for the magnitude of change described in percentage points (PP). Alpha was set at the $5 \%$ level. All analysis was undertaken using SPSS version 21 (IBM, Armonk, NY). 


\section{Results}

Patient acuity

The average number of patient-days for 2007-2012 in NICU was 4,610 (range 3,994 to 5,681). In PICU the average number of patient-days for 2007-2012 was 3,333 (range 3,050 to 3,516). The mean length of stay in NICU was 30.2 days $(\mathrm{SD} \pm 11.1)$ in 2007 and increased to 38.5 days $(\mathrm{SD} \pm 14.2)$ by $2012(P<0.0001)$. The mean length of stay in PICU was 28.3 days (SD \pm 19.0$)$ in 2007 and increased to $29.3(\mathrm{SD} \pm 13.3)$ by $2012(P<0.0001)$. In 2007 the ratio of nurse-to-patient admissions in NICU was $0.9: 1$ and steadily increased to reach $1.3: 1$ by 2012. In PICU the ratio of nurse-to-patient admissions remained at 1:1 with the exception of 2008 and 2009 when the ratio increased to $1.3: 1$ and 1.6:1 respectively before returning to $1: 1$ thereafter.

\section{Specific healthcare associated infections}

The rate of CAUTI in NICU remained stable between 2007 (9 per 1000 catheter-days, $95 \%$ CI $0.2-52$ ) and 2012 (4 per 1000 catheter-days, $95 \%$ CI 0.1-22) (IRR $0.43, P=0.670)$. In PICU the CAUTI rate remained stable between 2007 (8 per 1000 catheter-days, $95 \%$ CI 3-15) and 2012 (9 per 1000 catheter-day, $95 \%$ CI 5-15) (IRR 1.3, $P=0.622$ ).

The CLABSI rate in NICU did not change significantly $(P=1.0)$ between 2007 (0 per 1000 line-days, $95 \%$ CI $0-4$ ) and 2012 (0 per 1000 line-days, $95 \%$ CI 0-11). In PICU the CLABSI rate remained stable between 2007 ( 6 per 1000 catheter-days, $95 \%$ CI 3-13) and 2012 (8 per 1000 catheter-days, $95 \%$ CI 5-13) (IRR 1.2, $P=0.615$ ).

The rate of VAP in NICU remained unchanged between 2007 and 2012 ranging from 10 (95\% CI 7-14) per 1000 device-days in 2007 to 7 (95\% CI 4-10) per 1000 device-days (IRR $0.71, P=0.814$ ). In PICU the VAP rate remained unchanged from 9 (95\% CI 6-14) per 1000 device-days in 2007 to 11 (95\% CI 8-16) per 1000 device-days in 2012 (IRR 1.2, $P=0.412$ ).

\section{Total healthcare associated infections}

The rate of aggregated HAIs in NICU remained stable over the first six years of surveillance with the rate at 20 per 1000 patient-days in 2007 and 19 per 1000 patientdays in 2012 (IRR 0.93, $P=0.957$ ) (Table 1). The total HAI rate in PICU in 2007 was 39 per 1000 patient-days and declined significantly in 2010 (IRR $0.61, P<0.001$ ) and in 2012 (IRR 0.65, $P=0.002$ ) (Table 1).

\section{Multiple drug resistant microorganisms}

The rate of MDROs in NICU remained unchanged for A. baumanni starting at 2.26 per 1000 patient-days in 2007 and remained steady at 2.82 per 1000 patient-days in 2012 (IRR 0.8, 95 \% CI IRR 0.4-2, P=0.58), K.pneumonia at 1.13 and 0.7 per 1000 patient-days in 2007 and
Table 1 Healthcare associated infections for Neonatal and Pediatric ICUs between 2007 and 2012

\begin{tabular}{lll}
\hline Year & Neonatal ICU & Pediatric ICU \\
\hline 2012 & $19[16-23](108 / 5681)$ & $25[20-31](88 / 3460)$ \\
& IRR $=0.93,0.957$ & IRR $=0.65,0.002$ \\
2011 & $18[14-21](86 / 4792)$ & $24[19-29](83 / 3516)$ \\
& IRR $=0.87,0.925$ & IRR $=0.60,<0.001$ \\
2010 & $23[19-28](106 / 4522)$ & $24[19-30](83 / 3457)$ \\
& IRR $=1.10,0.925$ & IRR $=0.61,0.001$ \\
2009 & $20[16-24](84 / 4238)$ & $32[26-38](109 / 3411)$ \\
& IRR $=0.97,0.980$ & IRR $=0.81,0.117$ \\
2008 & $22[17-27](87 / 3994)$ & $38[31-45](116 / 3050)$ \\
& IRR $=1.1,0.967$ & IRR $=0.97,0.804$ \\
2007 & $20[17-25](91 / 4434)$ & $39[33-47](122 / 3106)$ \\
& IRR $=1$ (reference year) & IRR $=1$ (reference year) \\
\hline
\end{tabular}

Rate/1000 patient-days [95 \% Cl] (HAl/patient-days), Incidence rate ratio (IRR), $P$-value

2012 respectively (IRR $0.6,95 \%$ CI IRR $0.2-2.5, P=0.483$ ) and P.aeroginosa at 1.58 and 0.7 per 1000 patient-days in 2007 and 2012 respectively (IRR 0.4, 95\%CI IRR 0.1-1.4, $P=0.198)$. When all GNB were aggregated the rate in NICU from 2007 and 2012 (IRR 0.9, 95\%CI IRR 0.5-2, $P=0.824)$.

The rates in PICU remained unchanged for A. baumanni starting at 7.41 per 1000 patient-days in 2007 and remained steady at 5.49 per 1000 patient-days in 2012 (IRR 0.74, $95 \%$ CI IRR 0.5-1.4, $P=0.335$ ) and K.pneumonia also remained steady at 3.54 and 1.73 per 1000 patient-days between 2007 and 2012 respectively (IRR 0.5 , $95 \%$ CI IRR 0.2-1.4, $P=0.159$ ). P.aeroginosa decreased from 5.15 per 1000 patient-days in 2007 to 2.02 per 1000 patient-days by 2012 (IRR 0.4, $95 \%$ CI IRR $0.2-1 \quad P=0.039$ ). When all GNB MRDOs were aggregated the rate in PICU significantly decreased from 18.3 per 1000 patient-days in 2007 to 11.8 per 1000 patientdays by 2012 (IRR $0.7,95 \%$ CI IRR $0.5-1, P=0.033$ ).

The MRSA rate in NICU in 2007 was 0 per 1000 patient-days and remained stable with the exception of 2008 when 2 patients acquired MRSA infection ( 0.5 per 1000 patient-days, $P=1.0)$. The MRSA rate in PICU in 2007 was 2 per 1000 patient-days and dropped significantly to 0 per 1000 patient-days in $2012(P<0.05)$.

\section{Antimicrobial daily dose usage}

In NICU betalactam antibiotics (range 87 to $146 \mathrm{~g}$ ) were more commonly prescribed while carbapenems, aminoglycosides and glycopeptides were prescribed less frequently (Fig. 1).

By 2012 betalactam prescribing had decreased by $29 \%$ (122 g to $87 \mathrm{~g}$ ) and carbapenem consumption decreased by $42 \%$ (55.4 g to $32 \mathrm{~g}$ ). In PICU the most common 


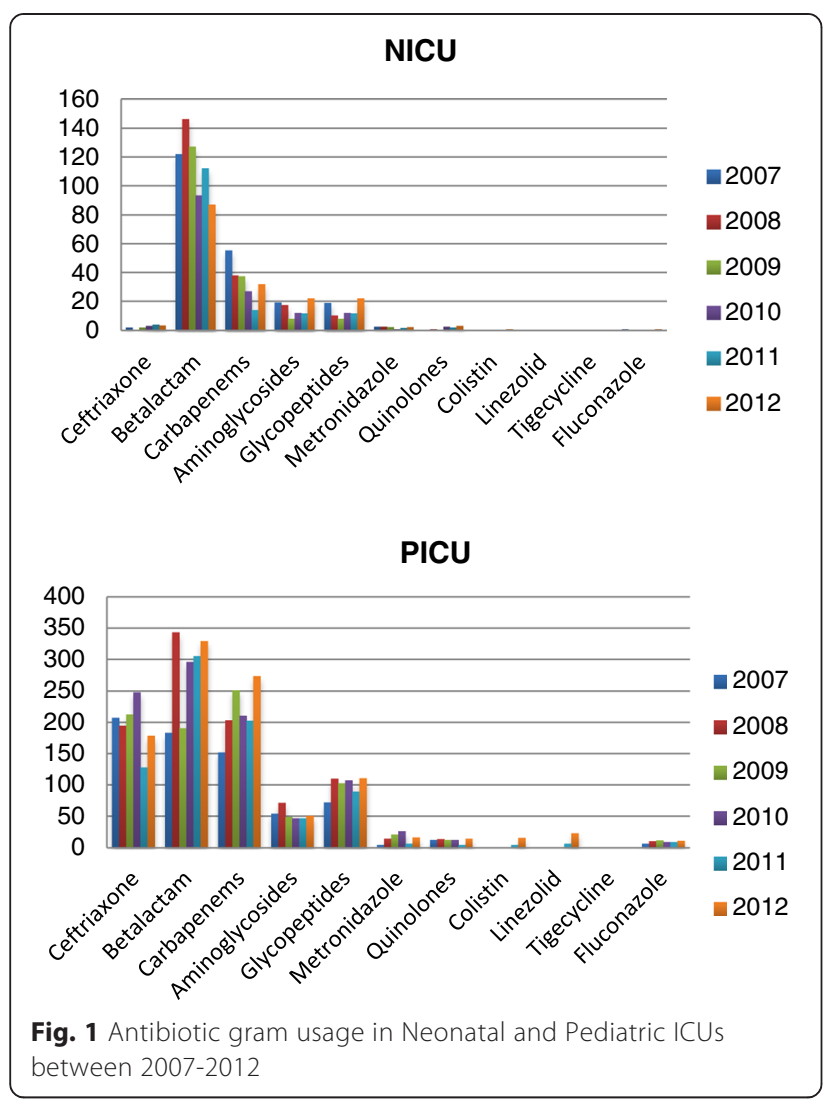

antibiotics were betalactams, carbapenems and ceftriaxone while aminoglycoside and glycopeptides were less common. In PICU by 2012, ceftriaxone usage had fallen $14 \%$ (207 g in 2007 to $178 \mathrm{~g}$ in 2012). Increases in usage in PICU were marked by an $80 \%$ increase in carbapenem usage (152 g in 2007 to $273 \mathrm{~g}$ in 2012) and $44 \%$ increase in betalactam usage (183 g in 2007 to $329 \mathrm{~g}$ in 2012). PICU and NICU infrequently prescribed metronidazole, quinolone, colistin, linezolid, tigecycline and fluconazole.

\section{Hand hygiene by moments}

Total compliance in hand hygiene significantly improved in both ICUs after the introduction in 2009 of the hand hygiene program (Tables 2 and 3). Compliance with moment 5 , after touching the patient surroundings, did not improved in either NICU or PICU. By 2012 compliance in the NICU increased significantly for moment 1 (IRR $1.5,65.6 \%, p=0.001$ ), moment 2 (IRR 2.0, $79 \%, p=0.001$ ) and moment 4 (IRR 1.4, $76 \%, p=0.006$ ) (Table 2). By 2012 compliance in PICU increased significantly for moment 1 (IRR 2.4, $50 \%, p<0.001$ ), moment 2 (IRR 4.6, $48 \%$, $p<0.001$ ) and moment 4 (IRR 1.5, $67 \%, p=0.008$ ) (Table 3). NICU had higher compliance than PICIU for moment 1 by 15 percentage points (PP) in 2012,31PP higher for moment 2 and 9PP higher for moment 4.
Total compliance in 2012 in NICU was 9PP higher than PICU and this was reflected in the ABHR usage in NICU that was three times higher than in PICU (Fig. 2).

\section{Discussion}

Intrinsic risk factors, such as an immature immune system and severity of illness, and extrinsic factors, such as poor health services infrastructure, invasive devices and the medication associated with these devices, increase the risk of HAI acquisition in patients admitted to NICU and PICU $[3,8]$. In low to middle income countries factors associated with the persistence of HAI in NICU and PICU are unlike those in high income countries, such as a lack of crucial elements of an infection control program, a legal framework for establishing and enforcing infection control programs and finances [2]. Other risk factors unique to low and middle income healthcare settings is overcrowded wards, poor nurse-to-patient ratio, patient malnutrition and severity of illness [2]. Since 2006 some of the essential components of the infection control programs in our ICUs are similar to high resourced countries and includes the development of infection control procedures, surveillance, education and monthly feedback to ICU staff on their HAI prevention performance. Yet, despite comparable device utilization rates to those reported in the USA the device-associated HAIs in both our ICUs were 2-20 times higher than the rates in USA [9]. Rosenthal et al. also reported a higher incidence of VAP in academic hospitals in low to middle income countries [3]. Smulders et al. demonstrated that infection prevention bundles are effective in neonatal and pediatric patients rate of CLABSI and VAP [10]. Yet, our intractable CAUTI, CLABSI and VAP rates remain unexplained and may be due to a lack of institutionalized compliance by our PICU or NICU with each of the bundle items. The drop in HAI is most likely in response to the aggregation of statistically rare events and not a result of infection prevention. Similarly, our inability to identify a significant change in MDROs in PICU except when we aggregated all GNB suggests that the sample size is possibly a factor. Our academic hospital receives patients transferred with severe illness. Important intrinsic factors contributing to HAI were not evaluated and compliance with prophylaxis was also not evaluated. However, poor antibiotic prescribing and poor hand hygiene compliance may be two important causal contributors.

Antibiotics are the most frequently used medicines in neonatal and pediatric ICUs. Only consumption in grams was measured so judgment of antibiotic overprescribing in our tertiary referral hospital with admissions and transfers of patients with severe disease can only be suggested from the trends in consumption. Although we expect antibiotics are commenced 
Table 2 Neonatal ICU Hand hygiene compliance by Moments between 2009 and 2012

\begin{tabular}{|c|c|c|c|c|c|c|}
\hline \multirow[t]{2}{*}{ Year } & \multicolumn{6}{|c|}{ Five Moments \% complied [95 \% Cl] (Observed/Total opportunities) Incidence Rate Ratio (IRR), $P$-value } \\
\hline & Moment 1 Before patient contact & Moment 2 Before a procedure & $\begin{array}{l}\text { Moment } 3 \text { After procedure/risk } \\
\text { of exposure to body fluid }\end{array}$ & Moment 4 After patient contact & $\begin{array}{l}\text { Moment } 5 \text { After contact } \\
\text { with patient environment }\end{array}$ & Total Moments \\
\hline 2012 & $\begin{array}{l}65.6[60.6-70.2](259 / 395) \\
\operatorname{IRR}=1.5,0.001\end{array}$ & $\begin{array}{l}78.7[68.7-86.6](70 / 89) \\
\operatorname{IRR}=2.0,0.001\end{array}$ & $\begin{array}{l}14.9[9.4-22.1](20 / 134) \\
\operatorname{IRR}=1.5,0.338\end{array}$ & $\begin{array}{l}76.4[71.7-80.6](281 / 368) \\
\operatorname{IRR}=1.4,0.006\end{array}$ & $\begin{array}{l}33.4[27.9-39.3](93 / 278) \\
\operatorname{IRR}=0.67,0.782\end{array}$ & $\begin{array}{l}57.2[54.4-59.9 \%](723 / 1264) \\
\operatorname{IRR}=2.2,<0.001\end{array}$ \\
\hline 2011 & $\begin{array}{l}62.5[58.1-66.7](318 / 509) \\
\operatorname{IRR}=1.4,0.004\end{array}$ & $\begin{array}{l}59.4[51.1-67.4](88 / 148) \\
\operatorname{IRR}=1.5,0.046\end{array}$ & $\begin{array}{l}25.8[18.4-34.4](32 / 124) \\
\operatorname{IRR}=2.6,0.016\end{array}$ & $\begin{array}{l}65.2[60.5-69.7](281 / 431) \\
\operatorname{IRR}=1.3,0.018\end{array}$ & $\begin{array}{l}55.7[47.8-63.4](93 / 167) \\
\operatorname{IRR}=0.59,0.731\end{array}$ & $\begin{array}{l}58.1[55.4-60.7](801 / 1379) \\
\operatorname{IRR}=2.9,<0.001\end{array}$ \\
\hline 2010 & $\begin{array}{l}57.0[53.3-60.7](414 / 726) \\
\operatorname{IRR}=1.3,0.031\end{array}$ & $\begin{array}{l}56.3[50.6-61.9](174 / 309) \\
\operatorname{IRR}=1.4,0.062\end{array}$ & $\begin{array}{l}17.4[12.8-22.9](41 / 235) \\
\operatorname{IRR}=1.7,0.150\end{array}$ & $\begin{array}{l}68.8[65.4-72.1](525 / 763) \\
\operatorname{IRR}=1.2,0.050\end{array}$ & $\begin{array}{l}37.7[30.3-45.5](63 / 167) \\
\operatorname{IRR}=1 \text { (reference year) }\end{array}$ & $\begin{array}{l}56.3[54.2-58.4](1217 / 2160) \\
\operatorname{IRR}=4.7,<0.001\end{array}$ \\
\hline 2009 & $\begin{array}{l}44.6[37.8-51.5](95 / 213) \\
\operatorname{IRR}=1 \text { (reference year) }\end{array}$ & $\begin{array}{l}39.0[28.0-50.7](30 / 77) \\
\mathbb{I R R}=1 \text { (reference year) }\end{array}$ & $\begin{array}{l}10.0[4.4-18.8](8 / 80) \\
\operatorname{IRR}=1 \text { (reference year) }\end{array}$ & $\begin{array}{l}56.1[48.8-63.1](111 / 198) \\
\text { IRR }=1 \text { (reference year) }\end{array}$ & - & $\begin{array}{l}43.0[38.8-47.1](244 / 568) \\
\text { IRR }=1 \text { (reference year) }\end{array}$ \\
\hline
\end{tabular}


Table 3 Pediatric ICU Hand hygiene compliance by Moments between 2009 and 2012

\begin{tabular}{|c|c|c|c|c|c|c|}
\hline \multirow[t]{2}{*}{ Year } & \multicolumn{6}{|c|}{ Five Moments \% complied [95\%CI] (Observed/Total opportunities) Incidence Rate Ratio (IRR), P-value } \\
\hline & $\begin{array}{l}\text { Moment } 1 \text { Before patient } \\
\text { contact }\end{array}$ & Moment 2 Before a procedure & $\begin{array}{l}\text { Moment } 3 \text { After procedure/risk } \\
\text { of exposure to body fluid }\end{array}$ & Moment 4 After patient contact & $\begin{array}{l}\text { Moment } 5 \text { After contact } \\
\text { with patient environment }\end{array}$ & Total Moments \\
\hline 2012 & $\begin{array}{l}50[45-55](231 / 461) \\
\mathrm{IRR}=2.4,<0.001\end{array}$ & $\begin{array}{l}48[40-55](88 / 185) \\
\operatorname{IRR}=4.6,<0.001\end{array}$ & $\begin{array}{l}28[23-33](97 / 345) \\
\operatorname{IRR}=4.2,0.001\end{array}$ & $\begin{array}{l}67.5[63-71](353 / 523) \\
\operatorname{IRR}=1.5,0.008\end{array}$ & $\begin{array}{l}43[39-47](251 / 585) \\
\text { IRR }=1.6,0.749\end{array}$ & $\begin{array}{l}49[46-51](1020 / 2099) \\
\operatorname{IRR}=1.9,<0.001\end{array}$ \\
\hline 2011 & $\begin{array}{l}46[41-52](179 / 385) \\
\operatorname{IRR}=2.2,0.001\end{array}$ & $\begin{array}{l}52[45-58](124 / 239) \\
\operatorname{IRR}=5.0,<0.001\end{array}$ & $\begin{array}{l}39[34-45](120 / 304) \\
\operatorname{IRR}=5.9,<0.001\end{array}$ & $\begin{array}{l}74[69.9-78.3](324 / 436) \\
\operatorname{IRR}=1.7,0.001\end{array}$ & $\begin{array}{l}48[42-54](139 / 291) \\
\operatorname{IRR}=1.4,0.807\end{array}$ & $\begin{array}{l}53[51-56](886 / 1655) \\
\operatorname{IRR}=2.4,<0.001\end{array}$ \\
\hline 2010 & $\begin{array}{l}43[39-47](315 / 733) \\
\operatorname{IRR}=2.0,0.002\end{array}$ & $\begin{array}{l}46[42-51](217 / 469) \\
\operatorname{IRR}=4.5,<0.001\end{array}$ & $\begin{array}{l}24[21-28](132 / 541) \\
\operatorname{IRR}=3.7,0.002\end{array}$ & $\begin{array}{l}67[63-71](467 / 696) \\
\operatorname{IRR}=1.5,0.008\end{array}$ & $\begin{array}{l}43[42-50](251 / 544) \\
\text { IRR }=1 \text { (reference year) }\end{array}$ & $\begin{array}{l}43[42-45](1296 / 2983) \\
\text { IRR }=2.1,<0.001\end{array}$ \\
\hline 2009 & $\begin{array}{l}21[14-31](21 / 99) \\
\text { IRR }=1 \text { (reference year) }\end{array}$ & $\begin{array}{l}10[4-20](7 / 68) \\
\text { IRR }=1 \text { (reference year) }\end{array}$ & $\begin{array}{l}7[2-14](6 / 90) \\
\operatorname{IRR}=1 \text { (reference year) }\end{array}$ & $\begin{array}{l}45[35-55](48 / 107) \\
\text { IRR }=1 \text { (reference year) }\end{array}$ & - & $\begin{array}{l}22[18-27](82 / 364) \\
\text { IRR }=1 \text { (reference year) }\end{array}$ \\
\hline
\end{tabular}




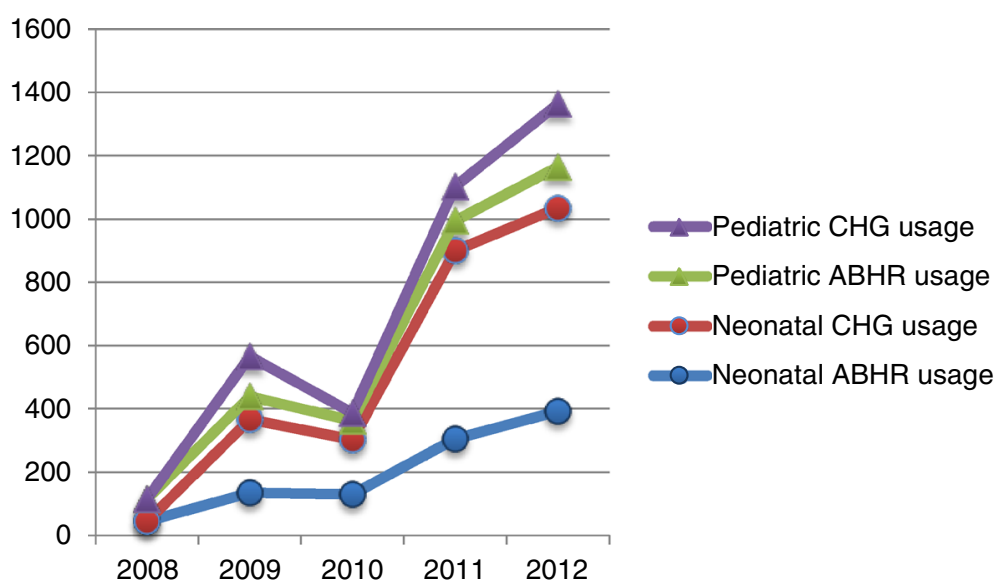

Fig. 2 Liter usage of Alcohol Based Hand Rub (ABHR) and Chlorhexidine (CGH) containing soap in Neonatal and Pediatric ICUs between $2008-2012$

empirically, consumption of the most commonly prescribed antibiotics in NICU appeared to have declined. The carbapenem resistant A.baumannii in PICU was endemic between 2009 and 2012 and unlike carbapenem usage globally [11] there was a conspicuous increase, $80 \%$, in carbapenem prescribing during this period. In NICU, staff carbapenem prescribing patterns decreased by $42 \%$ over the same period and may have been in response to a peak in carbapenem resistant A.baumannii in 2011. Colistin prescribing is rare in our ICUs unlike elsewhere [11]. The persistence of GNB MDROs, specifically A.baumannii, K.pneumonia and P. aeroginosa, and our patterns of repeated and prolonged prescribing need to be challenged to urgently address habitually poor prescribing practices by our ICU physicians [12-14]. Even in the absence of decolonization practices, such as chlorhexidine body wash [15], a modest improvement in our hand hygiene rates has kept the transmission of MRSA infections low [16]. We did not evaluate the association between antibiotic use and resistance patterns or reduction in infection due to change in antibiotic prescribing.

The introduction of a hand hygiene program in 2009 resulted in a 15PP improvement in total compliance by NICU staff and a 27PP improvement by PICU staff. Compliance with moment 1 , moment 2 and moment 4 improved 21PP, 40PP and 20PP respectively in NICU while poor compliance for moments 3 and 5 reduced the overall compliance rate. PICU staff performed moments 2,3 and 5 poorly while the improvements in Moments 1 and 4 by 29PP and 22PP respectively suggests a modest change in behavior. Glove use for moments 2 and 3 may explain our failure to achieve important behavioral change. However, even with the availability of $\mathrm{ABHR}$ and monthly compliance feedback to staff the final compliance in both ICUs was still low. The method of direct observation for monitoring hand hygiene compliance usually results in some degree of Hawthorne
Effect, therefore the usual compliance rates may in fact be lower than the rate we are reporting.

In Turkey, national infection control programs commenced nearly 50 years after other European countries. Despite our late start and limited resources, we have demonstrated that our surveillance activities have continued. Countries with limited resources have constraints in common such as, hospital infrastructure, overcrowding, understaffing, poor infection control program funding and adherence to guidelines. The barriers to further progression includes the continued high workload demonstrated by our very low nurse-to-patient ratio ranging $0.6: 1$ to 0.9:1 in PICU and 0.5:1 to 0.6:1 in NICU. High patient loads may be an important barrier to hand hygiene practice [17]. Low nurse-to-patient ratio significantly increases the risk for HAI with a much as $30 \%$ of HAI possibly being avoided when nurse-to-patient ratio is maintained at $>2.2$ nurses to one patient [18]. Two contributing factors to the endemic HAI rates and poor hand hygiene compliance in our ICUs may include nursing staff caring for $>1$ patient during night shifts and the prolonged night shift, routinely $16-\mathrm{h}$. These two factors have been found to adversely impact a clinician's ability to perform at their very best $[18,19]$. Innovative behavior change programs [20] may have a more immediate benefit on HAI rates given we may not be able to remedy the nurse-to-patient ratio in the near future. Acinetobacter species readily acquire resistance due to, in part, its excellent biofilm-producing ability that enables it to survive in hospital environments [21]. Any breakdown in environmental cleaning will contribute to a persistent endemicity, such as we have witnessed.

Our study provides an insight into the early phase of infection control programs in a middle-income country although we acknowledge that there are limitations to interpretations of our study that includes the collection of certain data retrospectively, absence of reasons for 
prescribing and patient demographic characteristics. Given any improvements in infrastructure will be slow to occur, a middle-income healthcare system such as ours needs to look towards a more effective use of existing resources, effecting governmental prioritization of HAI prevention programs and urgent changes to antibiotic prescribing practices.

\section{Competing interests}

The authors declare that they have no competing interests.

\section{Authors' contributions}

The concept and design of the study was by EA with input from MLM. Data were collected and validated by TO, CAK, SE. MLM analyzed, interpreted and developed the manuscript with EA participating in the interpretation and manuscript development. All authors read and approved the final manuscript.

\section{Authors' information}

EA is the Director, Department of Infectious Diseases and Clinical Microbiology, and the Infection Control Committee at Erciyes University Hospital, Kayseri, Turkey. TO, CAK, SE are the infection control team at Erciyes University Hospital. MLM is Professor of Epidemiology, Hospital Infection and Infectious Diseases Control at UNSW, Australia and collaborates with low and middle income countries on capacity building in infection control epidemiology.

\section{Author details}

${ }^{1}$ Faculty of Medicine, Department of Infectious Diseases and Clinical Microbiology, and Infection Control Committee, Erciyes University, Kayseri, Turkey. ${ }^{2}$ Faculty of Medicine, Faculty of Medicine, Infection Control Committee, Erciyes University, Kayseri, Turkey. ${ }^{3}$ School of Public Health and Community Medicine, UNSW Medicine, The University of New South Wales, Level 3 Samuels Building, Sydney, NSW 2052, Australia.

Received: 21 April 2015 Accepted: 13 August 2015

Published online: 25 August 2015

\section{References}

1. Porto JP, Mantese OC, Arantes A, Freitas C, Filho PPG, Ribas RM. Nosocomial infections in a pediatric intensive care unit of a developing country: NHSN surveillance. Rev Soc Bras Med Trop. 2012;45:475-9.

2. Zimmerman PA. Help or hindrance? Is current infection control advice applicable in low-and middle-income countries? A review of the literature. Am J Infect Control. 2007;35:494-500.

3. Rosenthal VD, Jarvis WR, Jamulitrat $\mathrm{S}$, Rodrigues $\mathrm{CP}$, Ramachandran $\mathrm{B}$, Duenas $L$, et al. Socioeconomic impact on device-associated infections in pediatric intensive care units of 16 limited-resource countries: International Nosocomial Infection Control Consortium findings. Pediatr Crit Care Med. 2012;13:399-406.

4. Horan TC, Andrus M, Dudeck MA. CDC/NHSN surveillance definition of health care-associated infection and criteria for specific types of infections in the acute care setting. Am J Infect Control. 2008;36:309-32.

5. World Bank. Gross national income country classification. World Bank. Available at: http://www.worldbank.org/en/country/turkey. Accessed August 18, 2015.

6. Alp E, Leblebicioğlu H, Doganay M, Voss A. Infection control practice in countries with limited resources. Ann Clin Microbiol Antimicrobials. 2011;10:36. doi:10.1186/1476-0711-10-36.

7. WHO guidelines on hand hygiene in health care 2009. http://www.who.int/ gpsc/information_centre/hand-hygiene-2009/en/. Accessed August 18, 2015.

8. Djordjevic ZM, Markovic-Denic L, Folic MM, Igrutinovic Z, Jankovic SM. Health care-acquired infections in neonatal intensive care units: risk factors and etiology. Am J Infect Control. 2015;43:86-8.

9. Dudeck MA, Horan TC, Peterson KD, Allen-Bridson K, Morrell G, Anttila A, et al. National Healthcare Safety Network report, data summary for 2011, device-associated module. Am J Infect Control. 2013:41:286-300

10. Smulders $C A$, van Gestel JPJ, Bos AP. Are central line bundles and ventilator bundles effective in critically ill neonates and children? Intensive Care Med. 2013;39:1352-8.
11. Meyer E, Gastmeier P, Deja M, Schwab F. Antibiotic consumption and resistance: Data from Europe and Germany. Int J Med Microb. 2013;303:388-95.

12. Paksu MS, Paksu S, Karadag A, Sensoy G, Asilioğlu N, Yildizdas D, et al. Old agent, new experience: colistin use in the paediatric Intensive Care Unit-a multicentre study. In J Antimicrob Agents. 2012;40:140-4.

13. Alp E, Percin D, Colakoglu S, Durmaz S, Kürkcü CA, Ekincioğu P, et al. Molecular characterization of carbapenem-resistant Klebsiella pneumoniae in a tertiary university hospital in Turkey. J Hosp Infect. 2013;84:178-80.

14. Alp E, Esel D, Yildiz O, Voss A, Melchers W, Doganay M. Genotypic analysis of Acinetobacter bloodstream infection isolates in a Turkish university hospital. Scan J Infect Dis. 2006;38:335-40.

15. Darouiche RO, Wall Jr MJ, Itani KMF, Otterson MF, Webb AL, Carrick MM, et al. Chlorhexidine- alcohol versus povidone-iodine for surgical-site antisepsis. N Engl J Med. 2010;362:18-26.

16. Allegranzi B, Gayet-Ageron A, Bengaly L, Damani N, McLaws ML, Moro ML, et al. Successful Implementation of the WHO Multimodal Hand Hygiene Promotion Strategy Across Facilities Worldwide. Lancet ID. 2013;13(10):843-51.

17. Alp E, Altun D, Cevahir F, Ersoy S, Cakir O, McLaws ML. Evaluation of the effectiveness of an infection control program in adult intensive care units: A report from a middle income country. Am J Infect Control. 2014;42(10):1056-61.

18. Hugonnet $S$, Chevrolet JC, Pittet D. The effect of workload on infection risk in critically ill patients. Crit Care Med. 2007;35:76-81.

19. Olds DM, Clarke SP. The Effect of Work Hours on Adverse Events and Errors in Health Care. J Safety Res. 2010;41(2):153-62.

20. Whitby M, Pessoa-Silva CL, McLaws ML, Allegranzi B, Sax H, Larson E, et al. Behavioural considerations for hand hygiene practices: the basic building blocks. J Hosp Infect. 2006;65(1):1-8.

21. Fournier PE, Richet $\mathrm{H}$. The epidemiology and control of Acinetobacter baumannii in health care facilities. Clin Infect Dis. 2006;42:692-9.

\section{Submit your next manuscript to BioMed Central and take full advantage of:}

- Convenient online submission

- Thorough peer review

- No space constraints or color figure charges

- Immediate publication on acceptance

- Inclusion in PubMed, CAS, Scopus and Google Scholar

- Research which is freely available for redistribution

Submit your manuscript at www.biomedcentral.com/submit
C BioMed Central 